ANÁLISIS DEL ESTRÉS Y NIVEL DE CONCENTRACIÓN DE ESTUDIANTES UNIVERSITARIOS DURANTE PERIODOS DE EXÁMENES.

\title{
Análisis del Estrés y Nivel de Concentración de Estudiantes Universitarios Durante Periodos de Exámenes
}

\section{Analysis of Stress and Concentration Level of University Students During Exam Periods}

\author{
Lobato Báez, Mariana*, Morales Rosales, Luis Alberto**,

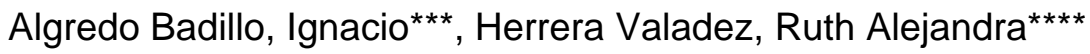

*Doctora en Planeación Estratégica y Dirección en Tecnologías. Instituto Tecnológico Superior de Libres. ORCID: https://orcid.org/0000-0002-2607-2032.

**Doctor en Ciencias Computacionales. Universidad Michoacana de San Nicolás de Hidalgo. Email: lamorales@conacyt.mx, ORCID: https://orcid.org/0000-0002-4753-9375.

***Doctor en Ciencias Computacionales. Instituto Nacional de Astrofísica Óptica y Electrónica. Email: ialgredo@conacyt.mx, ORCID: https://orcid.org/0000-0002-4753-9375.

****Ingeniera en Sistemas computacionales. Instituto Tecnológico Superior de Libres. Email: ale_herval@outlook.com, ORCID: https://orcid.org/0000-0002-1762-363X.

Correo para recibir correspondencia: mariana.lobato@upaep.edu.mx

Fecha de recibido: 7 de diciembre de 2020

Fecha de aceptación: 15 de febrero de 2021

LOBATO-BÁEZ M., MORALES-ROSALES L. A., ALGREDO-BADILLO I., HERRERA-VALADEZ R. A. 
ANÁLISIS DEL ESTRÉS Y NIVEL DE CONCENTRACIÓN DE ESTUDIANTES UNIVERSITARIOS DURANTE PERIODOS DE EXÁMENES.

\section{RESUMEN}

OBJETIVO: Identificar el nivel de estrés académico mediante el instrumento de medición SISCO y el nivel de concentración de los estudiantes.

MATERIAL Y MÉTODO: Se detectaron las frecuencias de ondas cerebrales de 122 estudiantes utilizando el dispositivo Muse Headband. Para medir el nivel de confiabilidad se empleó Alfa de Cronbach, obteniendo un valor de 0.83. Se realizó un análisis factorial, teniendo como resultado en la prueba de esfericidad de Bartlett de .000 y un valor de adecuación KMO (Medida Kaiser-Meyer-Olkin) de .783. Se utilizó el modelo de Regresión Lineal Múltiple para la identificación de asociación de variables.

RESULTADOS: Se evidencia una correlación de variables para el estrés académico relacionado a la fatiga crónica, aislamiento de los demás, tipos de trabajo que dejan los profesores, aumento o reducción de consumo de alimentos.

CONCLUSIONES: Los alumnos atribuyen el estrés académico a las evaluaciones, a la sobrecarga de tarea y trabajo, el tiempo limitado para la realización del mismo. Destacando como principales síntomas fueron: inquietud (incapacidad de relajarse y estar tranquilo); fatiga crónica (cansancio permanente); aumento o reducción de consumo de alimentos; ansiedad; angustia o depresión.

PALABRAS CLAVE: Estrés académico. Sisco. Stai. Regresión Lineal Múltiple. Análisis factorial.

\section{ABSTRACT}

OBJECTIVE: Identify the level of academic stress through the SISCO measurement instrument and the students' concentration level.

MATERIAL AND METHOD: The brain wave frequencies of 122 students were detected using the Muse Headband device. To measure the reliability level, Cronbach's Alpha was used, obtaining a value of 0.83 . A factorial analysis was performed, as a result in the Bartlett's sphericity test of .000 and a KMO (Kaiser-Meyer-Olkin measure) adequacy value of .783. The Multiple Linear Regression model was used to identify the association of variables. 
ANÁLISIS DEL ESTRÉS Y NIVEL DE CONCENTRACIÓN DE ESTUDIANTES UNIVERSITARIOS DURANTE PERIODOS DE EXÁMENES.

RESULTS: A correlation of variables is evidenced for academic stress related to chronic fatigue, isolation from others, types of work left by teachers, increase or decrease in food consumption.

CONCLUSIONS: the students attribute academic stress to evaluations, homework and work overload, and limited time to do it. Standing out as the main symptoms were: restlessness (inability to relax and be calm); chronic fatigue (permanent tiredness); increase or decrease in food consumption; anxiety; distress or depression.

KEY WORDS: Academic stress. Sisco. Stai. Multiple Linear Regression. Factorial analysis.

\section{INTRODUCCIÓN}

La inclusión del estrés como parte de la cotidianeidad del siglo XXI ha patentado su presencia en diferentes áreas de la vida (Águila, 2015). Como menciona Collazo (2011), el estrés es un problema social en la actualidad. Sin embargo, es un tema que no ha recibido suficiente atención en el ámbito de la investigación. Los alumnos de nivel superior tienen un ritmo acelerado, tareas, exámenes, compromisos, salidas con los amigos, son algunas actividades que los estudiantes universitarios realizan continuamente a lo largo del proceso de aprendizaje, lo que provoca una sobrecarga de sucesos, obteniendo como resultado el estrés, acción que repercute en el ámbito académico, físico y psicológico (Orlandini, 2012). Desde los grados preescolares hasta la educación universitaria de postgrado, cuando una persona está en un período de aprendizaje experimenta tensión y falta de nivel de concentración a esta se le denomina estrés académico y ocurre tanto en el estudio individual como en el aula. El estrés académico es un proceso que se presenta de manera descriptiva en tres momentos: primero: el alumno se ve sometido, a una serie de exigencias que, bajo la valoración del propio alumno son consideradas estresantes; segundo: estos estresores provocan un desequilibrio sistémico (situación estresante), que se manifiesta en una serie de síntomas (que son los indicadores del desequilibrio), y tercero: esta inestabilidad sistémica obliga al alumno a realizar acciones de adaptación para restaurar el equilibrio, lo que se conoce como estrategias de afrontamiento (Jerez y Oyarzo, 2015). El estrés académico es el malestar que el estudiante presenta debido a factores físicos, emocionales, ya sean de carácter interrelacional o ambientales que pueden ejercer una presión significativa en la competencia individual, para afrontar el contexto escolar en el rendimiento académico, habilidad meta cognitiva para resolver problemas, presentación de 
ANÁLISIS DEL ESTRÉS Y NIVEL DE CONCENTRACIÓN DE ESTUDIANTES UNIVERSITARIOS DURANTE PERIODOS DE EXÁMENES.

exámenes, relación con los compañeros y educadores, búsqueda de reconocimiento e identidad, habilidad para relacionar el componente teórico con la realidad específica abordada (Susana y Andrés, 2007). Las manifestaciones más comunes que provoca el estrés, a través de estados de constante alerta, inducen un sentimiento indefinido de inseguridad que se evidencia en lo orgánico: palpitaciones, náuseas, vómitos, tics localizados, temblor de pies y manos; y en lo psíquico: irritabilidad, temor, a veces angustia, inquietud interior, aspectos visibles en la conducta: bloqueo afectivo, alteraciones del lenguaje verbal, timidez o agresividad (Jaramillo y otros, 2008).

Para Jusset (2015), el estrés es un fenómeno adaptativo de los seres humanos que contribuye, en buena medida, a su supervivencia, a un adecuado rendimiento en sus actividades y a un desempeño eficaz en muchas esferas de la vida, la manera en que cada alumno transite su inicio en la etapa universitaria estará regulada por su adaptación a los factores estresores. Por lo tanto, aquellos alumnos que logren adaptarse a los cambios que conlleva el ámbito universitario aprenderán y se enriquecerán personalmente, tomando los cambios como un desafío (ya que es necesario experimentar cierto nivel de estrés para que se inicie una acción) pero, por el contrario, aquellos que no puedan "manejar" correctamente estas situaciones experimentarán insatisfacción personal y/o desgaste. Para Amanda (2004), el estrés a nivel mundial se ha convertido en un problema de salud pública de millones de personas, no solamente hay agentes nocivos que son productores de estrés; sino que, además, en el caso de las personas, las demandas sociales y las amenazas del entorno del individuo que requieren de capacidad de adaptación. Los factores de riesgo que contribuyen son los siguientes: la falta de tiempo y recursos económicos que hacen que los alumnos manifiestan síntomas transitorios de ansiedad, en respuestas a las exigencias académicas a las que se encuentran sometidos a realizarlas (Arrieta, Diaz, y Gonzalez, 2013). A nivel superior se observan manifestaciones de estrés debido a diferentes situaciones como la competencia, exceso de trabajo, fechas cercanas a exámenes parciales, conflictos en el salón de clases, expectativas no cumplidas, problemas familiares y las exigencias por parte de los docentes que los alumnos se tienen que enfrentar cada día derivando de ella falta de concentración y atención. La sociedad ha cambiado mucho últimamente y en que la juventud actual tiene una formación, básicamente los jóvenes viven inmersos en una sociedad audiovisual y tecnológica, donde internet va ganado terreno tanto a la hora de buscar información como en los momentos de ocio. Hoy en día, un joven pude recibir en un solo día mucha información que la podía recibir una persona del siglo XVIII durante toda su vida. Esta situación, lleva a que los estudiantes vivan inmersos en el 
ANÁLISIS DEL ESTRÉS Y NIVEL DE CONCENTRACIÓN DE ESTUDIANTES UNIVERSITARIOS DURANTE PERIODOS DE EXÁMENES.

mundo de la imagen y, por lo tanto, a tender respuestas emotivas más que reflexivas que emotivas, a desear las cosas inmediatamente y al gusto por el dinamismo y por el placer instantáneo (Bergés, 2008). En cambio, la escuela se mueve en el terreno de la palabra, que requiere de respuestas reflexivas y valora la paciencia y el esfuerzo. Por ello, es comprensible que ahora, la relación entre los profesores y alumnos no resulte fácil y debido a ello el nivel de angustia y de estrés es cada vez más alto en el ámbito educativo. La falta de atención y concentración por parte del alumnado se traduce en un comportamiento poco adecuado en clase y en malos resultados académicos. Con este contexto se recomiendan técnicas como la respiración consiente, la relajación, la visualización y la conciencia corporal, entre otras para intentar combatir el estrés y sustituirla por la cultura del bienestar (Teulé, 2008).

\section{MATERIAL Y MÉTODO}

\section{Muestra}

Se encuestaron a 122 alumnos de edades comprendidas entre los 18 y 29 años, de los cuales 60 eran hombres y 62 mujeres, distribuidos uniformemente en cuatro semestres del Instituto Tecnológico Superior de Libres, siendo una muestra representativa, a través de un muestreo intencional, de una Institución de Educación Superior que atienden a estudiantes de diferentes zonas tanto rurales como urbanas pertenecientes a la ciudad de Libres, Puebla, México.

\section{Instrumentos de investigación}

Para la presente investigación, se hizo uso del inventario de estrés académico SISCO, que tiene por objetivo reconocer las características de estrés que suelen acompañar a los estudiantes de educación media superior, superior y postgrado durante su estudio (Barraza, 2007). Para la aplicación la estructura fue conformada por 29 ítems distribuidos de la siguiente manera:

- Ocho ítems que, en un escalamiento tipo Lickert de cinco valores categoriales (nunca, rara vez, algunas veces, casi siempre y siempre) permiten identificar la frecuencia con que se presentan los síntomas o reacciones al estímulo estresor.

- Seis ítems que, en un escalamiento tipo Lickert de cinco valores categoriales (nunca, rara vez, algunas veces, casi siempre y siempre) permiten identificar las reacciones físicas. 
ANÁLISIS DEL ESTRÉS Y NIVEL DE CONCENTRACIÓN DE ESTUDIANTES UNIVERSITARIOS DURANTE PERIODOS DE EXÁMENES.

- Cinco ítems que, en un escalamiento tipo Lickert de cinco valores categoriales (nunca, rara vez, algunas veces, casi siempre y siempre) permiten identificar las reacciones psicológicas.

- Cuatro ítems que, en un escalamiento tipo Lickert de cinco valores categoriales (nunca, rara vez, algunas veces, casi siempre y siempre) permiten identificar las reacciones comportamentales.

- Seis ítems que, en un escalamiento tipo Lickert de cinco valores categoriales (nunca, rara vez, algunas veces, casi siempre y siempre) permiten identificar el afrontamiento.

Para identificar el nivel de concentración de los estudiantes se hizo uso Muse Headband con 1 ítems que mide la frecuencia cerebral permitiendo determinar si la concentración del estudiante es activa, medianamente activa, neutral, medianamente neutral o se encuentra en calma.

\section{Recolección de datos}

El inventario SISCO fue aplicado a 122 estudiantes, permitiendo medir estresores, reacciones físicas, reacciones psicológicas, reacciones comportamentales y afrontamiento. Las preguntas fueron cerradas, teniendo opciones de respuestas que fueron previamente delimitadas (Tabla 1).

\section{Tabla 1}

Respuestas del inventario de SISCO

\begin{tabular}{lll}
\hline$\square$ & 1 & Nunca \\
$\square$ & 2 & Rara vez \\
$\square$ & 3 & Algunas veces \\
4 & 4 & Casi siempre \\
$\square$ & 5 & Siempre \\
\hline
\end{tabular}

Fuente: Elaborado con datos recolectados.

Para el desarrollo de esta investigación, se empleó Muse Headband, permitiendo recolectar el nivel de concentración de 122 estudiantes teniendo como respuesta los siguientes indicativos que se muestran en la Tabla 2. El escenario para la recopilación de los datos de las ondas cerebrales (EEG) de los estudiantes del ITS Libres fue una sala de espera que se encuentra instalada en el edificio de Investigación y Posgrado. El momento en que se colocaron los sensores del dispositivo Muse Headband fue posterior a la semana de exámenes programados 
ANÁLISIS DEL ESTRÉS Y NIVEL DE CONCENTRACIÓN DE ESTUDIANTES UNIVERSITARIOS DURANTE PERIODOS DE EXÁMENES.

para el primer parcial de los estudiantes. La duración de la observación de las señales EEG fue de 5 minutos por cada estudiante. Este ejercicio trata de caracterizar si el nivel de concentración al finalizar el examen es igual o diferente para los estudiantes de las distintas carreras del ITS Libres.

\section{Tabla 2}

Indicativos de Muse Headband

Activo Indicando que el estudiante tiene una mete totalmente distraída donde la atención fluctúa.

Mediana mente Indicando que el estudiante tiene una mete distraída pero la atención fluctúa.

activo

Neutral Indica que la atención del estudiante se encuentra en total reposos, la atención no fluctúa, pero tampoco hay un enfoque profundo presente.

Mediana mente Indica que la atención del estudiante se encuentra en reposos, la atención no fluctúa, pero neutral tampoco hay un enfoque profundo presente.

Calma Indica los momentos en los que los estudiantes están completamente concentrados.

Fuente: Elaboración propia.

En el proceso de recolección de datos (Figura 1), se observa que el 12\% de los estudiantes indicaron no tener estrés, el 29\% describe que rara vez, el 35\% describe que algunas veces el $18 \%$ casi siempre y finalmente el $6 \%$ siempre. Referente a las reacciones físicas el $25 \%$ de los estudiantes indicaron no tener raciones físicas, el 33\% describe que rara vez, el 28\% indicaron que algunas veces el $12 \%$ casi siempre y finalmente el $2 \%$ siempre. En las reacciones psicológicas se puede observar que el $17 \%$ de los estudiantes indicaron no tener reacciones, el $47 \%$ describe que rara vez, el $24 \%$ indicaron que algunas veces, el $8 \%$ casi siempre y finalmente, el $4 \%$ siempre. En las reacciones comportamentales se observa que el $11 \%$ de los estudiantes indicaron no tenerlas, el 36\% describe que rara vez, el 37\% indicaron que algunas veces el $10 \%$ casi siempre y finalmente el $6 \%$ siempre. En la forma de afrontar el estrés se identificó que el $12 \%$ de los estudiantes indicaron no tienen afrontamiento, el $29 \%$ describe que rara vez, el $38 \%$ indicaron que algunas veces el 16\% casi siempre y finalmente, el 5\% siempre.

Referente al nivel de concentración el 5\% de los estudiantes tiene una mente activa que indica una mente distraída donde la atención fluctúa, mientras que el 29\% tiene una mente medianamente activa, el 32\% tiene un estado de mente neutral que indica que la atención no fluctúa, pero tampoco hay un enfoque profundo, el 10\% tiene una mente medianamente neutral y finalmente, el $24 \%$ de los estudiantes indico tener una concentración profunda y reparadora. 
ANÁLISIS DEL ESTRÉS Y NIVEL DE CONCENTRACIÓN DE ESTUDIANTES UNIVERSITARIOS DURANTE PERIODOS DE EXÁMENES.

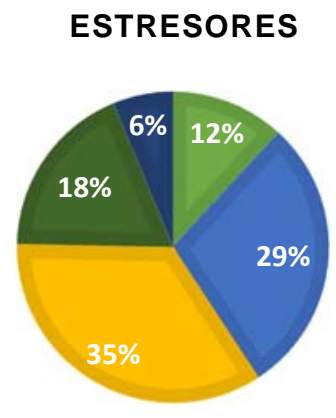

REACCIONES
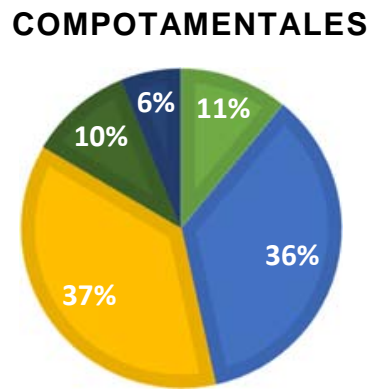

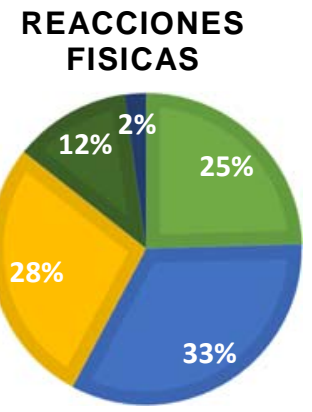

AFRONTAMIENTO

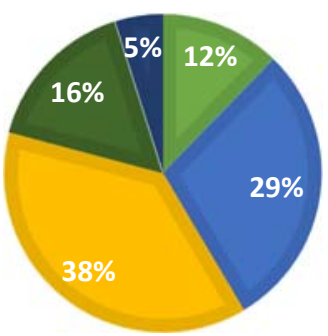

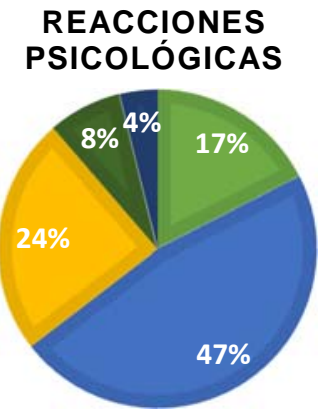

NIVEL DE CONCENTRACIÓN

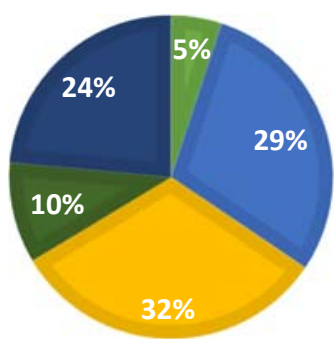

Figura 1. Porcentajes de estrés y nivel de concentración de los estudiantes. Fuente: Elaboración propia.

\section{Confiabilidad del instrumento de medición}

Para identificar la confiabilidad, se observaron 122 casos, donde se presentan los casos válidos, excluidos y totales, así como sus valores conceptuales. Asimismo, se observa un análisis con Alfa de Cronbach, obteniendo un valor de .882 de confianza.

\section{Tabla 3}

Procesamiento de casos

\begin{tabular}{ccc}
\hline Casos & $\mathrm{N}$ & $\%$ \\
\hline Válido & 122 & 100.0 \\
Excluido & 0 & .0 \\
Total & 122 & 100.0
\end{tabular}

Fuente: Elaboración propia.

\section{Tabla 4}

Estadísticas de fiabilidad

Alfa de Cronbach $\quad$ Alfa de Cronbach basada en $\quad \mathrm{N}$ de elementos
elementos estandarizados

$\begin{array}{lll}.882 & .894 & 30\end{array}$

Fuente: Elaboración propia. 
ANÁLISIS DEL ESTRÉS Y NIVEL DE CONCENTRACIÓN DE ESTUDIANTES UNIVERSITARIOS DURANTE PERIODOS DE EXÁMENES.

\section{Análisis factorial}

Para el análisis factorial, se realizó la prueba de esfericidad de Bartlett, obteniendo un valor de .000 y un valor de adecuación como se muestra en la Tabla 5 y de la muestra en KMO (Medida Kaiser-Meyer-Olkin) de .783.

\section{Tabla 5}

KMO (Medida Kaiser-Meyer-Olkin)

\begin{tabular}{llll}
\hline \multicolumn{2}{l}{ Medida Kaiser-Meyer-Olkin de adecuación de muestro } & .783 \\
\hline Prueba de esfericidad de & Aprox. Chi-cuadrado & 1514.378 \\
Bartlett & & gl & 465 \\
& & Sig. & .000
\end{tabular}

Fuente: Elaboración propia.

\section{RESULTADOS}

\section{Matriz de componentes rotada del análisis factorial}

Se utilizó el análisis factorial Varimax considerando seis factoriales (F1, F2, F3, F4, F5, F6). Cada factor representa la suma de las respuestas a las preguntas $(\mathrm{P} 1+\mathrm{P} 2+\ldots+\mathrm{P} 30)$ con pesos específicos asociados, y la combinación lineal de las variables. Se analizó cada dimensión extraída e identificando las variables que tienen elevada correlación con un factor. En la Tabla 6, se observan cada uno de los factores en donde, el F1 tiene relación con aspectos de reacciones físicas como trastornos del sueño, fatiga crónica, dolores de cabeza o migraña, problemas de digestión, somnolencia, problemas de concentración, F2 tienen reacciones comportamentales como son conflictos o tendencias a polemizar o discutir, aislamiento con los demás, desgano para realizar las labores, aumento o reducción de alimentos, F3 tiene relación con la competencia con los compañeros del grupo, sobrecarga de tareas y trabajos escolares, personalidad y carácter del profesor, evaluaciones de los profesores, F4 tiene relación con afrontamiento como la búsqueda de información sobre la situación que preocupa y verbalización de la situación que preocupa, F5 tiene relación con estresores en donde es la competencia con los compañeros de grupo y con afrontamiento búsqueda de información de la situación que preocupa, F6 tiene relación con afrontamiento elaboración de un plan y ejecución 
ANÁLISIS DEL ESTRÉS Y NIVEL DE CONCENTRACIÓN DE ESTUDIANTES UNIVERSITARIOS DURANTE PERIODOS DE EXÁMENES.

de sus tareas. Referente al nivel de concentración, se puede observar en (V31) que los valores son bajos en la correlación con el estrés.

Al realizar la correlación agrupada para el nivel de concentración (Tabla 7) que se consideraron solo dos factores destacados con la correlación de la frecuencia cerebral y la carrera.

\section{Tabla 6}

Estadísticas de fiabilidad estrés académico

\begin{tabular}{|c|c|c|c|c|c|c|}
\hline Pregunta / Factor & $\mathrm{F} 1$ & $\mathrm{~F} 2$ & F3 & $\mathrm{F} 4$ & F5 & F6 \\
\hline V1 & 0.211 & 0.217 & 0.206 & -0.073 & 0.707 & 0.015 \\
\hline V2 & 0.155 & 0.187 & 0.661 & -0.078 & 0.159 & 0.204 \\
\hline V3 & 0.022 & 0.113 & 0.726 & 0.007 & 0.055 & -0.070 \\
\hline V4 & 0.177 & 0.168 & 0.716 & 0.153 & -0.009 & 0.092 \\
\hline V5 & 0.173 & 0.053 & 0.755 & 0.168 & 0.061 & 0.132 \\
\hline V6 & 0.064 & 0.127 & 0.455 & -0.097 & 0.379 & 0.096 \\
\hline V7 & 0.090 & 0.039 & 0.365 & -0.042 & 0.493 & -0.052 \\
\hline V8 & 0.144 & 0.065 & 0.672 & 0.108 & 0.087 & -0.022 \\
\hline V9 & 0.640 & 0.058 & 0.123 & 0.029 & 0.216 & 0.268 \\
\hline V10 & 0.778 & 0.250 & 0.182 & 0.107 & 0.040 & -0.094 \\
\hline V11 & 0.640 & 0.199 & -0.013 & 0.160 & 0.171 & -0.138 \\
\hline V12 & 0.735 & 0.074 & 0.127 & 0.005 & 0.166 & -0.028 \\
\hline V13 & 0.357 & 0.314 & 0.173 & 0.074 & 0.267 & 0.046 \\
\hline V14 & 0.633 & 0.183 & 0.363 & -0.157 & -0.108 & 0.042 \\
\hline V15 & 0.360 & 0.481 & 0.288 & 0.127 & 0.270 & -0.082 \\
\hline V16 & 0.449 & 0.582 & 0.127 & 0.039 & 0.121 & -0.023 \\
\hline V17 & 0.549 & 0.505 & 0.262 & 0.057 & -0.037 & -0.048 \\
\hline V18 & -0.105 & 0.447 & -0.156 & -0.319 & 0.326 & 0.154 \\
\hline V19 & 0.266 & 0.677 & 0.105 & 0.040 & 0.197 & 0.042 \\
\hline V20 & 0.016 & 0.690 & 0.028 & 0.234 & 0.098 & 0.152 \\
\hline V21 & 0.122 & 0.758 & 0.141 & 0.011 & -0.017 & -0.220 \\
\hline V22 & 0.475 & 0.550 & 0.205 & -0.016 & -0.191 & -0.049 \\
\hline V23 & 0.215 & 0.575 & 0.307 & -0.139 & -0.120 & 0.248 \\
\hline V24 & 0.023 & -0.009 & 0.131 & 0.062 & -0.114 & 0.896 \\
\hline V25 & -0.120 & 0.018 & 0.150 & 0.181 & 0.194 & 0.702 \\
\hline V26 & -0.128 & 0.045 & -0.072 & 0.424 & 0.097 & 0.272 \\
\hline V27 & 0.036 & -0.031 & 0.200 & 0.019 & 0.049 & 0.038 \\
\hline V28 & 0.262 & -0.254 & -0.016 & 0.277 & 0.523 & -0.004 \\
\hline V29 & 0.144 & -0.032 & 0.079 & 0.787 & -0.122 & 0.148 \\
\hline V30 & 0.038 & 0.180 & 0.164 & 0.797 & 0.083 & 0.018 \\
\hline V31 & -0.020 & -0.006 & 0.062 & 0.014 & -0.006 & -0.110 \\
\hline
\end{tabular}

Fuente: Elaboración propia. 
ANÁLISIS DEL ESTRÉS Y NIVEL DE CONCENTRACIÓN DE ESTUDIANTES UNIVERSITARIOS DURANTE PERIODOS DE EXÁMENES.

Tabla 7

Estadísticas de fiabilidad nivel de concentración

\begin{tabular}{llc}
\hline Pregunta / Factor & F1 & F2 \\
\hline Carrera & .623 & -.526 \\
Semestre & .605 & .412 \\
Frecuencia Cerebral & .081 & .833 \\
Sexo & -.782 & -.014 \\
\hline
\end{tabular}

Fuente: Elaboración propia.

\section{Regresión lineal múltiple}

Para identificar la correlación de variables que influyen estrés académico y el nivel de concentración se utilizó las respuestas de 122 estudiantes al aplicar el inventario de medición SISCO y la diadema Muse Headband. Al utilizar Regresión Lineal Múltiple, mostrado en la tabla 8, el modelo determinó un valor de 48.7\%. Por otro lado, el error típico de la estimación (raíz cuadrada de la varianza no explicada) resulta ser de 0.878 .

\section{Tabla 8}

Correlación de variables

\begin{tabular}{ll}
\hline Modelo & 1 \\
\hline $\mathrm{R}$ & .698 \\
R Cuadrada & .487 \\
R Cuadrado Ajustado & .325 \\
Error estándar de la Estimación & .792 \\
Cambio en R cuadrado & .878
\end{tabular}

Fuente: Elaboración propia.

\section{Coeficientes de Regresión Lineal Múltiple}

La utilización del modelo de Regresión Lineal Múltiple, permitió identificar los factores que influyen en el estrés académico detonando la sobrecarga de tareas y trabajos escolares, dolores de cabeza o migraña, somnolencia o mayor necesidad de dormir, sentimientos de depresión y tristeza, aumento de irritabilidad, aumento o reducción del consumo de alimentos, para la concentración cerebral detonaron el semestre y la carrera. En la Tabla 9, se observan 
ANÁLISIS DEL ESTRÉS Y NIVEL DE CONCENTRACIÓN DE ESTUDIANTES UNIVERSITARIOS DURANTE PERIODOS DE EXÁMENES.

los valores altos discriminando aquellos valores bajos (no influyentes) arrojados por el modelo de Regresión Lineal Múltiple.

\section{Tabla 9}

Coeficientes no estandarizados de Regresión Lineal Múltiple

\begin{tabular}{cccccc}
\hline Pregunta / Coeficiente & B & Desv. Error & Beta & t & Sig. \\
\hline P2 & 0.163 & 0.099 & 0.173 & 1.652 & 0.102 \\
P10 & 0.165 & 0.127 & 0.169 & 1.303 & 0.196 \\
P13 & 0.157 & 0.103 & 0.172 & 1.522 & 0.131 \\
P15 & 0.171 & 0.108 & 0.174 & 1.584 & 0.117 \\
P18 & 0.173 & 0.147 & 0.103 & 1.179 & 0.241 \\
P23 & 0.154 & 0.133 & 0.147 & 1.154 & 0.251 \\
Pregunta / Coeficiente & B & Desv. Error & Beta & $\mathrm{t}$ & Sig. \\
P2 & 0.163 & 0.099 & 0.173 & 1.652 & 0.102 \\
P5 & 0.154 & 0.100 & 0.175 & 1.534 & 0.129 \\
P10 & 0.165 & 0.127 & 0.169 & 1.303 & 0.196 \\
P13 & 0.157 & 0.103 & 0.172 & 1.522 & 0.131 \\
P15 & 0.171 & 0.108 & 0.174 & 1.584 & 0.117 \\
P16 & 0.148 & 0.116 & 0.174 & 1.269 & 0.208
\end{tabular}

Fuente: Elaboración propia.

Para los coeficientes estandarizados, el modelo de Regresión Lineal Múltiple (Tabla 9), refleja los factores de sobrecarga de tareas y trabajos escolares, tipos de trabajos que dejan los profesores, fatiga crónica cansancio permanente, mayor necesidad de dormir, sentimientos de depresión y tristeza, ansiedad angustia y depresión.

En cuanto a la concentración, detonó que dos de los factores que influyen para la concentración son el semestre y la carrera. 
ANÁLISIS DEL ESTRÉS Y NIVEL DE CONCENTRACIÓN DE ESTUDIANTES UNIVERSITARIOS DURANTE PERIODOS DE EXÁMENES.

Tabla 10

Coeficientes estandarizados de Regresión Lineal Múltiple

\begin{tabular}{lccccc}
\hline Pregunta / Coeficiente & B & Desv. Error & Beta & t & Sig. \\
\hline Sexo & -0.16 & .108 & -0.14 & -.150 & .881 \\
Carrera & 0.117 & .062 & -.179 & -1.898 & .060 \\
Semestre & 0.101 & .084 & .110 & 1.201 & .232
\end{tabular}

Fuente: Elaboración propia.

La Tabla 10, muestra el modelo de Regresión Lineal Múltiple, en donde el valor $t$ reflejo la significancia estadística de los coeficientes en donde se observan los valores máximos detonantes de estrés académico, teniendo como factores influyentes la sobrecarga de tareas y trabajos escolares, tipo de trabajos que piden los profesores, mayor necesidad de dormir, sentimientos de depresión y tristeza, verbalización de la situación que preocupa, para la concentración detono el semestre que están cursando estudiante.

\section{Tabla 11}

Significación estadística de los coeficientes

\begin{tabular}{cccccc}
\hline Pregunta / Coeficiente & $\mathrm{B}$ & Desv. Error & Beta & $\mathrm{t}$ & Sig. \\
\hline P2 & 0.163 & 0.099 & 0.173 & 1.652 & 0.102 \\
P5 & 0.154 & 0.100 & 0.175 & 1.534 & 0.129 \\
P13 & 0.157 & 0.103 & 0.172 & 1.522 & 0.131 \\
P15 & 0.171 & 0.108 & 0.174 & 1.584 & 0.117 \\
P26 & 0.130 & 0.092 & 0.151 & 1.423 & 0.158 \\
P29 & 0.127 & 0.078 & 0.146 & 1.627 & 0.107
\end{tabular}

Fuente: Elaboración propia.

\section{Análisis de resultados}

El estrés académico representa un problema grave en el nivel superior, y por otro lado puede ser considerado como un área de oportunidad para que técnicas de aprendizaje sean fortalecidas con el fin de guiar a los estudiantes a enfrentar los futuros problemas del ámbito laboral. Asimismo, se presentó un análisis factorial en conjunto Regresión Lineal Múltiple, que permite analizar elementos, tales como: estresores, reacciones físicas, psicológicas, comportamentales y el afrontamiento ante el estrés presentados por los estudiantes de nivel 
ANÁLISIS DEL ESTRÉS Y NIVEL DE CONCENTRACIÓN DE ESTUDIANTES UNIVERSITARIOS DURANTE PERIODOS DE EXÁMENES.

superior. Se observó, que el nivel de concentración de los estudiantes si es distinguible por cada carrera, el sexto y el semestre que cursan. Los resultados reflejan que se requiere de una evaluación continua del nivel comportamental para generar estrategias que disminuyan el estrés. Se requiere un análisis posterior de correlación entre estos resultados y el rendimiento académico para distinguir entre un estrés que permite generar una mayor productividad y aquel que refleja un perjuicio para el estudiante durante el periodo de exámenes.

\section{CONCLUSIONES}

Se identificó el nivel de estrés académico mediante el instrumento de medición SISCO y el nivel de concentración de los estudiantes con base en la detección de las frecuencias de ondas cerebrales, concluyendo que los educandos utilizan con mayor frecuencia las estrategias de afrontamiento ante vivencias que demandan su entorno educativo. Enfatizando las estrategias de afrontamiento más utilizadas por los estudiantes son: concentrase en resolver la situación que más le preocupa, fijarse o tratar de obtener lo positivo de la situación que le preocupa, elaboración de un plan y ejecución de tareas, búsqueda de ayuda sobre la situación, y elogios, entre otros. Por otra parte, se concluye que los alumnos atribuyen el estrés académico a las evaluaciones, a la sobrecarga de tarea y trabajo, el tiempo limitado para la realización del mismo. Destacando como principales síntomas fueron: inquietud (incapacidad de relajarse y estar tranquilo); fatiga crónica (cansancio permanente); aumento o reducción de consumo de alimentos; ansiedad; angustia o depresión.

\section{REFERENCIAS BIBLIOGRÁFICAS}

Águila, B. A., Castillo, M. C., De la Guardia, R. M. y Achon, Z. N. (2015). Estrés académico. Edumecentro, 7(2), 12.

Amanda, G. (2004). Organización del trabajo y del estrés. Organización mundial para la salud, 3-7.

Bergés, E. y Teulé, A. R. (2008). Cómo ser docente y no morir en el intento: Técnicas de concentración y relajación en el aula. Vol. 13. Graó.

Collazo, C. A. (2011). El estrés académico: una revisión crítica del concepto desde las ciencias de la educación. Revista Electrónica de Psicología Iztacala, 14(2), 1-14. 
ANÁLISIS DEL ESTRÉS Y NIVEL DE CONCENTRACIÓN DE ESTUDIANTES UNIVERSITARIOS DURANTE PERIODOS DE EXÁMENES.

Díaz, E. S. M., \& Gómez, D. A. D. (2007). Una aproximación psicosocial al estrés escolar. Educación y educadores, 10(2), 11-22.

Jaramillo, G., Caro, H., Parra, Z., Bedoya, J., Pabón, É., \& Mejía, M. (2008). Dispositivos desencadenantes de estrés y ansiedad en estudiantes de Odontología de la Universidad de Antioquia. Revista Facultad de Odontología Universidad de Antioquia, 20(1).

Jerez Mendoza, M., \& Oyarzo Barría, C. (2015). Estrés académico en estudiantes del Departamento de Salud de la Universidad de Los Lagos Osorno. Revista chilena de neuro-psiquiatría, 53(3), 149-157.

Jusset, H. S. (2015). Estres académico en los estudiantes que cursan el primer año del ámbito universitario. Ciencias de la salud, 84.

Orlandini, A. (2012). El estrés: Qué es y cómo evitarlo. Fondo de cultura económica.

Selye, H. (2013). The evolution of the stress concept american scientist. 61: 692.

Vergara, K. A., Cárdenas, S. D., \& Martínez, F. G. (2013). Síntomas de depresión, ansiedad y estrés en estudiantes de odontología: prevalencia y factores relacionados. Revista Colombiana de Psiquiatría, 42(2), 173-181. 